\title{
Applying Soft Computing Techniques to Optimise a Dental Milling Process
}

\author{
Vicente Vera ${ }^{1}$, Emilio Corchado ${ }^{2,3}$, Raquel Redondo ${ }^{4}$, Javier Sedano ${ }^{5}$, Álvaro E. García ${ }^{1}$ \\ ${ }^{1}$ Facultad de Odontología, UCM, Madrid, Spain. \{viventevera, aegarcia\}@odon.ucm.es \\ ${ }^{2}$ Dept. de Informática y Automática, Universidad de Salamanca, Spain. escorchado@usal.es \\ ${ }^{3}$ Visiting Professor at VŠB-TUO, VŠB-Technical University of Ostrava, Czech Republic. escorchado@usal.es \\ ${ }^{4}$ Department of Civil Engineering, University of Burgos, Burgos, Spain. rredondo@ubu.es \\ ${ }^{5}$ Dept. of AI \&Applied Electronics, Castilla y León Technological Institute, Burgos, Spain. javier.sedano@itcl.es
}

\begin{abstract}
This study presents a novel soft computing procedure based on the application of artificial neural networks, genetic algorithms and identification systems, which makes it possible to optimise the implementation conditions in the manufacturing process of high precision parts, including finishing precision, while saving both time and financial costs and/or energy. This novel intelligent procedure is based on the following phases. Firstly, a neural model extracts the internal structure and the relevant features of the data set representing the system. Secondly, the dynamic system performance of different variables is specifically modelled using a supervised neural model and identification techniques. This constitutes the model for the fitness function of the production process, using relevant features of the data set. Finally, a genetic algorithm is used to optimise the machine parameters from a non parametric fitness function. The novel proposed approach was tested under real dental milling processes using a highprecision machining centre with five axes, requiring high finishing precision of measures in micrometers with a large number of process factors to analyse. The results of the experiment, which validate the performance of the proposed approach, are presented in this study.
\end{abstract}

Keywords. Soft Computing, Unsupervised Learning, Genetic Algorithm, Identification Systems, Optimisation, Dental Milling Process

\section{Introduction}

It is becoming increasingly necessary to have intelligent software tools to optimise tasks associated with modelling industrial processes, especially those associated with high precision finishing, such as the dental milling process.

The optimisation of machine parameters in the fabrication process could potentially improve the flexibility of the process, the adjustments of machine parameters, research in new materials, and its implementation in the fabrication process. It also improves some future designs. Presently, this is achieved with the help of experts (Research and development units in companies work to adjust parameters from the experimental design by carrying out a number of machine trials based on their own experiences). Machine parameter optimisation in the fabrication process includes the development of models to assess the behaviour of the variables in the process and to find the fitness function that can be optimised. The machine parameter optimisation should help the experts in better understanding the production process itself in order to produce products using new materials in a short period of time.

The application of the optimisation process in the field of Medical Therapeutics (Odonto-Stomatology), a booming industry, is both novel and economically advantageous [1, 2, 3, 4, 5, 6]. Improved processing and optimisation of parameters such as processing time, accuracy, etc., for the development of pieces (such as dental-oral prostheses to perform partial crowns, inlays, onlays, etc. with application for rehabilitation and oral-dental restoration) are to the focus of rigorous studies today. The optimisation process of machine parameters, for example the time parameter, permits significant economic savings due to the high number of dental pieces produced daily by the same high-precision dental milling machine centre. This could significantly help to increase a company's efficiency, and substantially contribute to 
cost reductions in the preparation and setting of the machines processes. Another example is the marginal adjustment of a dental prosthesis to the remaining tooth structure (dentine and enamel), thus avoiding tissue invasion and/or unprepared anatomical areas. This is the goal of any dental treatment, since the success now lies in the adjustment of approximately 15-25 micrometers between the prosthesis and the remaining structures. This would enhance and prevent the filtering of germs and oral fluids that within the short or medium term will lead to treatment failure.

For many years the traditional process of making and preparing dental structures has involved the use of wax, followed by a process called "Lost Wax" [7].

This is a valid methodology, but could interfere with the preparation of a series of variables that are not securely controlled. In fact, there have been cases in which the prosthesis has not fit the tooth structure correctly, resulting in short or medium term tooth decay and failure of the treatment. The optimal outcome would be the so-called passive adjustment, i.e. adjusted between 15-25 micrometers which would lead to success in dental restorative treatment.

Because of these and other reasons it is currently of great interest to optimise processes $[8,9]$ related to the preparation of dental prostheses (dental structures of materials such as cobalt chromium, titanium, ceramics and/or resin) characterised by a high precision of adjustment in micrometers.

Artificial Intelligence [10], in conjunction with optimisation and identification algorithms [11, 12], is a very appropriate technology for addressing the development of such intelligent tools. Nevertheless, the variable and parameter setting processes are a well-known problem that has not yet been fully resolved. Several different techniques have been proposed in literature. In [13] a Taguchi orthogonal array is used to optimise the effect of injection parameters; in [14] the influence of ultrasonic machining operating parameters is studied using Taguchi and the F-test method; [15] explores different ways of improving the quality of the KrF excimer laser micromachining of metal using the orthogonal array-based experimental design method. Conventional methods can be greatly improved through the application of soft computing techniques [16].

The novel method proposed in this research is a three-step procedure based on several soft computing techniques as artificial neural networks (ANN) [17, 18] and genetic algorithms (GA) [19, 20, 21]. Firstly, the dataset is analysed using statistical and projection methods such as Principal Component Analysis (PCA) [22, 23, 24] and Cooperative Maximum-Likelihood Hebbian Learning (CMLHL) [25] to extract the dataset structure and to perform feature selection to establish whether the data set is sufficiently informative. This means that if the initial collected data set, once analysed shows a certain degree of clustering, it can be seen as a sign of a representative data set (there are no problems related to any sensor when collecting the information, and the process is well defined by the data set).The subsequent steps of the process can then be applied, in which the most representative features are identified and used. A model is generated during the modelling stage to estimate, in this case, the production time errors by modelling techniques. As previously explained, this study is interested in decreasing the production time. Finally, the ANN model obtained in the last step is used as a fitness function to be optimised in the genetic algorithm.

This paper is organised as follows. Section 2 introduces the unsupervised neural models for analysing the datasets. Section 3 presents the system identification techniques used in the system modelling. Section 4 introduces the applied GA. Section 5 describes the case study: a real dental milling process. Section 6 presents the optimising of a dental milling process. The final section presents the different models that are used to solve the high precision dental milling optimisation case study. At the end, the conclusions are set out and some comments on future research lines are outlined.

\section{Data Structure Analysis using Connectionist Techniques}

Soft Computing [10, 26, 27, 28, 29, 30] is a set of several technologies whose aim is to solve inexact and complex problems [31, 32]. It investigates, simulates, and analyses very complex issues and phenomena in order to solve real-world problems [33, 34]. Soft Computing has been successfully applied in many different fields as, for example, feature selection $[17,18]$.

In this study, an extension of a neural PCA version [22, 23, 24] and other Exploratory Projection Pursuit $[35,36,37,38]$ extensions are used to select the most relevant input features in the data set and to study its internal structure.

Feature Selection [39, 40, 41] and extraction [42, 43, 44, 45] entails feature construction, space dimensionality reduction, sparse representations and feature selection among others. They are all 
commonly used pre-processing tools in machine learning tasks, which include pattern recognition. Although researchers have grappled with such problems for many years, renewed interest has recently surfaced in feature extraction.

The feature selection approach in this study is based on the issue of dimension reduction. Initially, some projection methods such as PCA [22, 23, 24], MLHL [36] and CMLHL [25, 46, 47] are applied. Their first step is to analyse the internal structure of a representative data set from a case study. If after applying these models, a clear internal structure can be identified, this means that the data recorded is informative enough. Otherwise, further data must be properly collected [8, 9].

\subsection{Principal Component Analysis}

Principal Component Analysis (PCA) originated in work by Pearson [22], and independently by Hotelling [23], is a statistical method describing multivariate data set variations in term of uncorrelated variables, each of which is a linear combination of the original variables. Its main goal is to derive new variables, in decreasing order of importance, which are linear combinations of the original variables and are uncorrelated with each other.

From a geometrical point of view, PCA can be defined as a rotation of the axes of the original coordinate system to a new set of orthogonal axes that are ordered in terms of the amount of variation of the original data that they account for. PCA aims to find that orthogonal basis which maximises the data's variance for a given dimensionality of basis.

Using PCA, it is possible to find a smaller group of underlying variables that describe the data. PCA has been the most frequently reported linear operation involving unsupervised learning for data compression and feature selection [24].

\subsection{A Neural Implementation of Exploratory Projection Pursuit}

The standard statistical method of EPP [25, 37, 38], provides a linear projection of a data set, but it projects the data onto a set of basic vectors which best reveal the interesting structure in data. Interestingness is usually defined in terms of how far the distribution is from the Gaussian distribution [48].

One neural implementation of EPP is Maximum Likelihood Hebbian Learning (MLHL) [36]. It identifies interestingness by maximizing the probability of the residuals under specific probability density functions that are non-Gaussian.

An extended version of this model is the Cooperative Maximum Likelihood Hebbian Learning (CMLHL) [25, 49] model. CMLHL is based on MLHL [36] adding lateral connections [25, 49], which have been derived from the Rectified Gaussian Distribution [48]. The resultant net can find the independent factors of a data set but does so in a way that captures some type of global ordering in the data set.

Considering an $\mathrm{N}$-dimensional input vector $(x)$, and an M-dimensional output vector $(y)$, with $\mathrm{W}_{\mathrm{ij}}$ being the weight (linking input $\mathrm{j}$ to output $\mathrm{i}$ ), then CMLHL can be expressed [49] as:

Feed-forward step:

$$
y_{i}=\sum_{j=1}^{\mathbf{N}} W_{i j} x_{j}, \forall i
$$

Lateral activation passing:

$$
y_{i}(t+1)=\left[y_{i}(t)+\tau(b-A y)\right]^{+}
$$

Feedback step:

$$
e_{j}=x_{j}-\sum_{i=1}^{M} W_{i j} y_{i}, \forall j
$$

Weight change:

$$
\Delta W_{i j}=\eta \cdot y_{i} \cdot \operatorname{sign}\left(e_{j}\right)\left|e_{j}\right|^{p-1}
$$

Where: $\eta$ is the learning rate, [ ]+ is a rectification necessary to ensure that the $y$-values remain within the positive quadrant, $\tau$ is the "strength" of the lateral connections, $b$ is the bias parameter, $p$ is a 
parameter related to the energy function $[25,36]$ and $A$ is the symmetric matrix used to modify the response to the data [25]. The effect of this matrix is based on the relation between the distances separating the output neurons.

\section{System Identification and Modelling}

System identification (SI) [11, 26] aims to obtain mathematical models to estimate the behaviours of a physical process whose dynamic equations are unknown. The identification criterion consists in evaluating the group of candidate models that best describes the dataset gathered for the experiment; that is, given a certain model $M\left(\theta_{*}\right)$, its prediction error may be defined as in Eq. (5), where $y(t)$ is the real output and $\hat{y}\left(t \mid \theta_{*}\right)$ is the prediction of this. The goal is to obtain a model that meets the following premise [11]: a good model is one that makes good predictions and which produces small errors when the observed data is applied.

Classic SI refers to the parametrical literature, which has its origin in linear system analysis [12]. Nevertheless, increased computational capability and the availability of soft computing techniques have widened research into SI. ANNs are one of the most interesting soft computing paradigms used in SI. When using ANN, the purpose of an identification process is to determine the weight matrix based on the observations $Z^{t}$, so as to obtain the relationships between the network nodes. The supervised learning algorithm is then applied to find the estimator $\theta$, so as to obtain the identification criterion. In this case, the minimization of the mean square error criterion as defined in Eq. (6) and Eq. (7) is used. The iterative minimization scheme is defined in Eq. (8), where $\theta(t)$ is the estimated parametrical vector, $f(t)$ represents the search direction and $\mu(t)$ the step size.

The SI procedure comprises several steps: the selection of the models and their structure, the learning methods, the identification and optimisation criteria and the validation method [11, 12, 50, 51, 52]. Validation ensures that the selected model meets the necessary conditions for estimation and prediction. Typically, validation is carried out using three different methods: the residual analysis $\varepsilon(t, \hat{\theta}(t)$ ) (by means of a correlation test between inputs, their residuals and their combinations); the mean squared error (MSE) and the generalization error value (normalised sum of squared errors (NSSE), and finally a graphical comparison between the desired outputs and the model outcomes through simulation [8, 9, 12].

$$
\begin{gathered}
\varepsilon\left(t, \theta_{*}\right)=y(t)-\hat{y}\left(t \mid \theta_{*}\right) \\
V_{N}\left(\theta, z^{t}\right)=\frac{1}{N} \sum_{t=1}^{N}[y(t)-\hat{y}(t \mid \theta)]^{\mathrm{T}}[y(t)-\hat{y}(t \mid \theta)] \\
\hat{\theta}=\underset{\theta}{\arg \min } V_{N}\left(\theta, Z^{t}\right) \\
\theta(t+1)=\theta(t)+\mu(t) f(t)
\end{gathered}
$$

\section{Genetic Algorithms for System Optimisation}

Metaheuristic algorithms $[53,54]$ are considered a computational method that optimises a problem by iteratively trying to improve a candidate solution with regards to a given measure of quality. Metaheuristics are powerful strategies that can efficiently detect high-quality (near optimal) solutions to complex optimization problems within reasonable running time.

Metaheuristics make few or no assumptions about the problem being optimised and can search very large spaces of candidate solutions. Among these algorithms, there are two well-known types: the genetic algorithms [55, 56, 57], and the simulated annealing algorithm [58, 59]; other methods can be: Tabu search [60,61] and ant colony optimisation [62].

GA $[19,20,21,63,64]$ are a type heuristic search that mimics the process of natural evolution (Darwin's theory about evolution). This heuristic is routinely used to generate useful solutions to optimisation and search problems. It solves both constrained and unconstrained optimisation problems.

Genetic algorithms find $\left(x_{i}, \ldots, x_{n}\right)$ such that $f\left(x_{i}, \ldots, x_{n}\right)$ will be maximum or minimum. The functions are shown from Eq. (9) to Eq. (11), where $f(x)$ is the fitness function, $c(x)$ represents inequality constraints, 
$c e q(x)$ represents the equality constraints, $m$ is the number of nonlinear inequality constraints and $m t$ is the total number of nonlinear constraints:

$$
\begin{gathered}
\text { Min or Max } f(x) \\
c_{i}(x) \leq a_{i} ; i=1, \ldots, m \\
\operatorname{ceq}_{i}(x)=b_{i} ; \quad i=m+1, \ldots, m t
\end{gathered}
$$

\section{A Real Case Study: Optimising a Dental Milling Process}

The acronym CAD/CAM (Computer Aided Design / Computer Aided Manufacturing) refers to a production technique that combines computer skills, which are then applied in both the design and manufacturing of pieces. Originally applied in the field of engineering, its use is now widespread, extending to many other areas.

In the field of dentistry, CAD/CAM systems are used primarily to manufacture fixed prosthetic restorations such as inlays, veneers and crowns. During the last decade, the technological evolution of these systems has provided restoration alternatives to rehabilitate teeth deficiencies, using different materials such as porcelain, composite and metal blocks, which previously could not be processed due to technical limitations [65].

There is currently an increased interest in manufacturing pillars and in making the structure of the prosthesis implant using CAD/CAM technology [1]. There are several reasons for this increase. First, the structure of the prosthetic implant is constructed from a solid block of material. With this specific production technique, the material is more homogeneous and contains high mechanical properties. Second, the inaccuracies are reduced since the processes of waxing, coating and casting no longer exist [66]. The prosthetic implants drawn up by CAD/CAM technology present more passive adjustment than the cast structures [2, 3, 4].

Since 1971 a marginal fit of less than 120 micrometers in tooth-supported restorations [67] has been regarded as clinically acceptable. In the prosthetic implant, the tolerable discrepancy between the implant abutment and the prosthetic supra-structure can be variable. A mismatch that does not exceed 30 micrometers can be tolerated [68], although Branemark et al. declare that the discrepancy must never exceed 10 micrometers [69]. However there is a consensus that the lack of passive adjustment acts as a causal factor in many technical complications such as loosening and/or fractures in the metal structure, retaining screws, abutments and ceramic or acrylic [70].

To perform any one of the many treatments allowed by this systematic approach, the CAD/CAM systems consist of the following stages of processing:

1. Digitization of the substrate that will make the restoration. It can be taken directly, optically in the patient's mouth, or extra-orally, after making a conventional impression, and emptied in a plaster cast.

2. Computer aided design. This is done by the specific software for each system, designing the prosthetic cap structure or the final restoration. This step is not done in those cases where the digitization is from the scanning of the structure to be obtained.

3. Once the design of the structure is finished, the next step is to manufacture the structure, which is achieved by applying the third stage of processing, the machining or CAM phase [5].

The operating system is accomplished through computer numerical control (CNC). The data obtained using the CAD-software are converted into "commands" which are "read" by the milling machine and then translated into drilling steps.

This multidisciplinary study uses a 5-axis milling (latest generation) device. In addition to controlling movement between the tool and the piece in three axes, this 5-axis machine can also control both the rotation of the piece in two axes, one perpendicular to the axis of the tool and the other parallel to it, and the rotation of the piece on a horizontal axis with the inclination of the tool around an axis perpendicular to the former. The advantages of 5-axis machines are numerous: it allows complete multilateral machining in a single cycle, which implies a reduction of non-productive time and eliminates the lack of precision arising from the multiple ties of the piece. It also allows better access to restricted areas difficult to reach. The angle adjustment can be freely defined. Another advantage is that it is possible to use shorter and more rigid tools, which results in improved surface finish. 
This multidisciplinary research describes the way in which a soft computing system can be applied to optimise the data gathered by means of a Machining Milling Center of HERMLE type-C 20 U (iTNC 530), with swivelling rotary $(280 \mathrm{~mm})$, with a control system using high precision drills and bits (Fig. 4 to Fig. 6), by optimising the time error detection for manufacturing dental metal. Fig. 1 to Fig. 3 show the metal pieces manufacturing process using a dynamic high-precision machining centre with five axes.

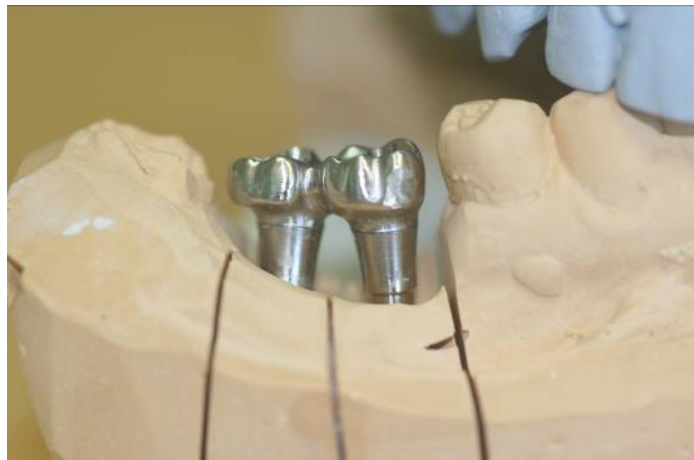

Fig. 1 Metal milled cobalt-chromium consists of a bridge with two lower molars

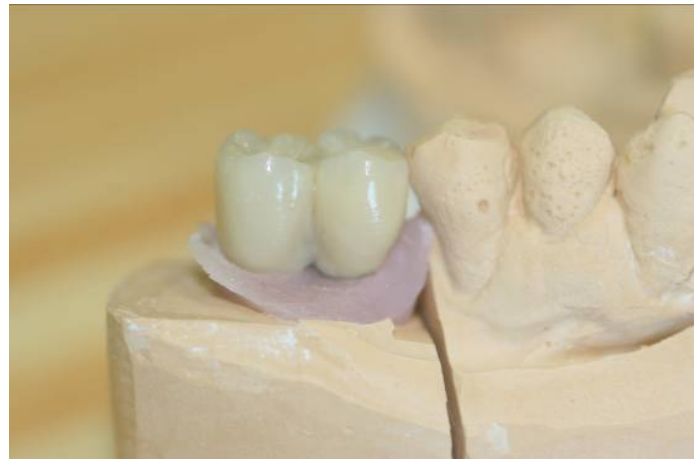

Fig. 3 Finished metal-porcelain bridgework

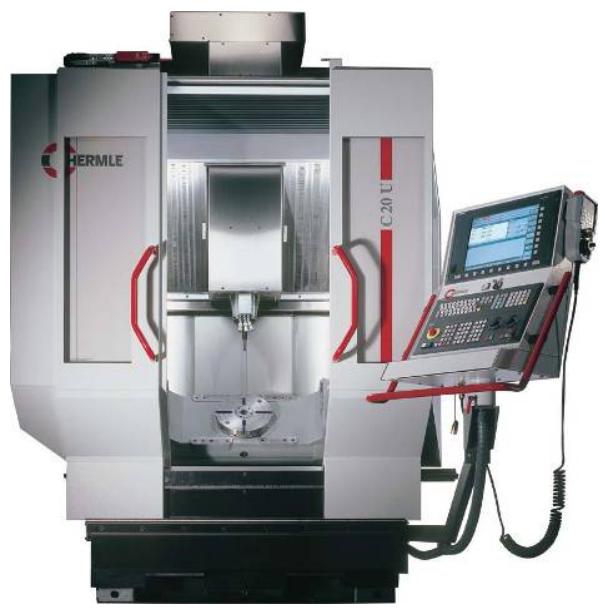

Fig. 5 Machining/ Milling Center of HERMLE type-C $20 \mathrm{U}$ (iTNC 530), with swivelling rotary (280 mm), with a control system using drills and bits of high precision

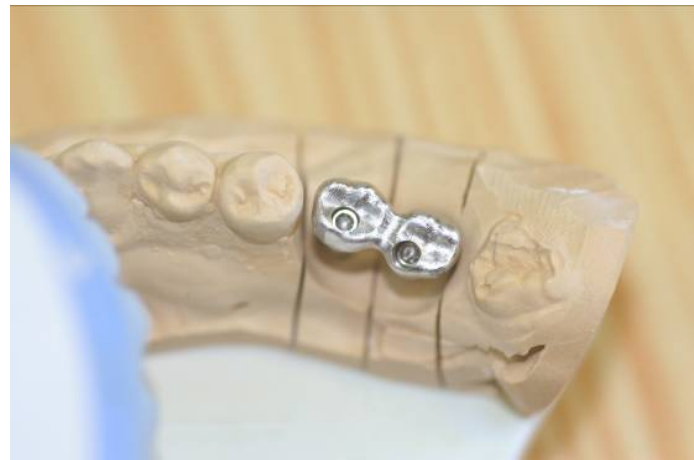

Fig. 2 Occlusal view of milled cobalt-chromium consists of a bridge with two lower molars

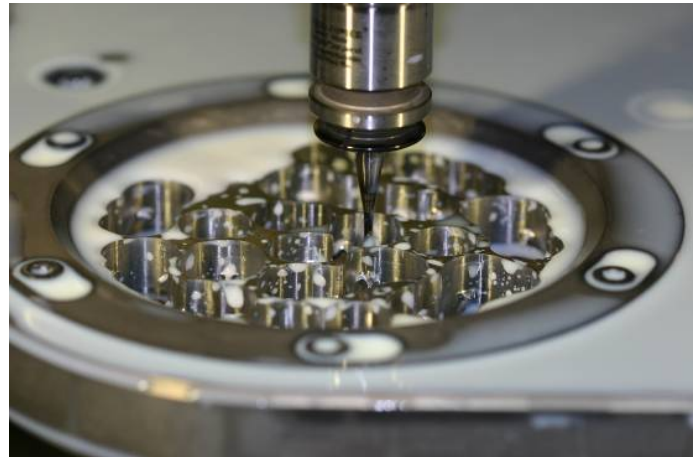

Fig. 4 Milling of cobalt-chromium specimens

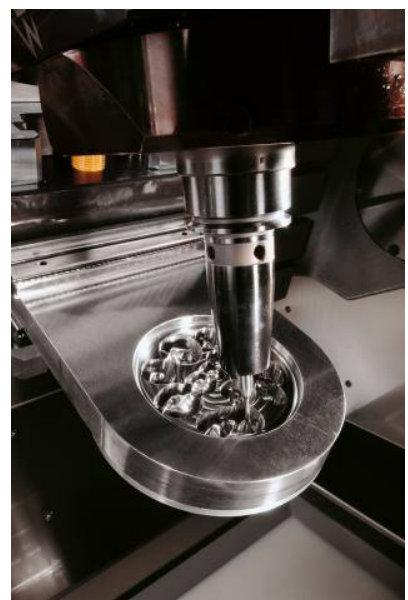

Fig. 6 Metal pieces manufactured by a dynamic highprecision machining centre with five axes

The case study is described by an initial data set of 98 samples obtained by the dental scanner in the manufacturing of dental pieces with different tool types (plane, toric, spherical and drill) and characterized by 8 input variables (Tool, Radius, Revolutions, Feed rate X, Y and Z, Thickness, Initial 
Temperature) and 1 output variable (Time Error for manufacturing) as shown in Table 1. Time error for manufacturing is the difference between the estimated time by the machine itself and real production time (negative values indicate that real time exceeds estimated time).

\begin{tabular}{lc}
\hline Variable (Units) & Range of values \\
\hline Type of tool & Plane, toric, spherical and drill \\
Radius (mm.) & 0.25 to 1.5 \\
Revolutions per minute (RPM) & 7,500 to 38,000 \\
Feed rate X (mm. by minute) & 0 to 3,000 \\
Feed rate Y (mm. by minute) & 0 to 3,000 \\
Feed rate Z (mm. by minute) & 50 to 2,000 \\
Thickness (mm.) & 10 to 18 \\
Temperature $\left({ }^{\circ} \mathrm{C}\right)$ & 24.1 to 31 \\
Real time of work (s) & 6 to 1,794 \\
Time errors for manufacturing (s) & -28 to -255 \\
\hline
\end{tabular}

Table 1 Values of each variable used in the process

\section{A Novel Soft Computing Procedure to Optimise a Dental Milling Process}

The manufacturing of dental pieces process optimisation in terms of time errors, based on the optimisation of the system behaviour, is carried out within the framework of this study by means of an ANN estimated model. The time error parameter is chosen as an important factor in this process (in terms of economical benefits for the company) as an example to show the potential of this novel soft computing proposal.

\subsection{Identification of the Relevant Features}

Firstly, the dental manufacturing process is parameterised and its dynamic performance in normal operation is obtained by the real process of manufacturing dental pieces. Then, the gathered data is processed using projection models based on the analysis of parameters as the variance [22, 23, 24] or the kurtosis as CMLHL [25, 46, 47, 49]. This is done to identify internal data set structures in order to analyse whether the data set is sufficiently representative and to identify the most relevant features in the second step.

\subsection{Modelling and optimisation of a normal dental milling operation}

Once the relevant variables and their transformations have been extracted from the production data, then a model capable of fitting the normal manufacturing operation must be obtained. This is done to identify bias in the estimated production time. The different model learning methods used in this study were implemented in Matlab®C [71]. The model structures were analysed in order to obtain the models that best suited the dataset. Since the number of examples was somewhat small; a 10-fold cross-validation schema was selected. The number of samples is low as they were obtained during the real process, delaying the company timing. The final model is obtained using the entire data set.

Moreover, several different indexes were used to validate the models $[8,9]$ such as the percentage representation of the estimated model; the graphical representation for the prediction $\left(\hat{y}_{1}(t \mid m)\right)$ versus the measured output $\left(y_{1}(t)\right)$; the loss function or error function $(\mathrm{V})$ and the generalization error value.

The percentage representation of the estimated model is calculated as the normalised mean error for the prediction (FIT1, FIT) using the validation data set and the complete data set, respectively. The loss function or error function (V) is the numeric value of the mean square error (MSE) that is computed using the estimation data set; the generalisation error value is the numeric value of the normalised sum of square errors (NSSE) that is computed using the validation data set. Finally, is calculated the variance of the mean square errors $(\lambda)[34,72]$.

Once the model for the time error in the manufacturing of dental pieces is selected, this model is used as a fitness function in GA's in order to obtain the best optimisation of the time errors. This optimisation process begins with a set of solutions called population (chromosomes). Each individual in the population 
is then evaluated by the fitness function obtained in the last step (ANN model of the manufacturing system). GA and the different types of genetic operators (selection, crossover and mutation) used in this study were implemented in Matlab@ [73]. The complete novel soft computing procedure is showed in Fig. 7.

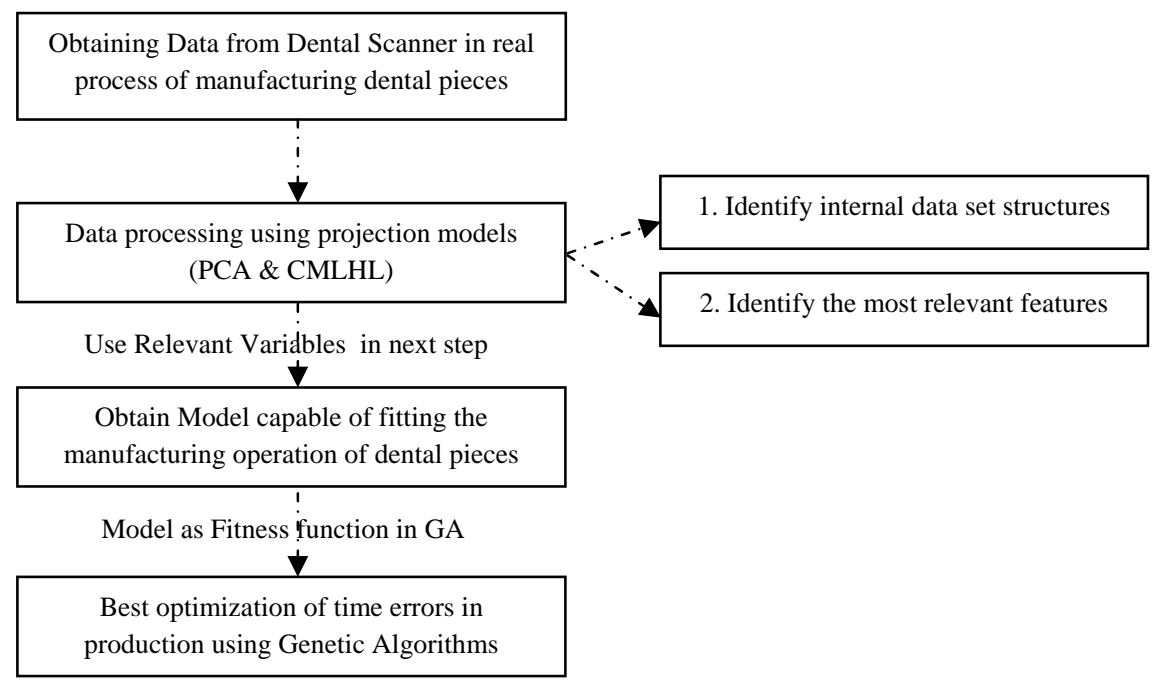

Fig. 7 A novel soft computing procedure to optimise a Dental Milling Process

\section{Results}

This case study initially analysed the data set in order to obtain the variables/characteristics that are most closely related to manufacturing time errors.

In the first step, several unsupervised models were applied for the sake of comparison. In this case a neural version of PCA and CMLHL were applied as powerful techniques for identifying internal dataset structures. The axes forming the projections (Fig. 8.a and Fig. 8.b) represent combinations of the variables contained in the original datasets. In the case of PCA, the model is looking for those directions with the biggest variance, while CMLHL is looking for the kurtosis (directions which are as little Gaussian as possible) [25, 36].

As may be seen in Fig. 8, PCA (Fig. 8.a) and CMLHL (Fig. 8.b), both methods found a clear internal structure in the dataset by identifying several clusters (see Table 2 and Table 3). Both also identified revolutions and radius as relevant variables. It is clear that CMLHL provides a more sparse representation than the PCA, and that CMLHL projections provide more clear information identifying parameters such as temperature and time error as other important variables.

An analysis of the results obtained with the CMLHL model (Fig. 8.b) leads to the conclusion that this method has identified several different clusters ordered by radius, revolutions and temperature. Inside each cluster, there are further classifications by 'time error' and the dataset can be said to have an interesting internal structure based on the clusters identified. 


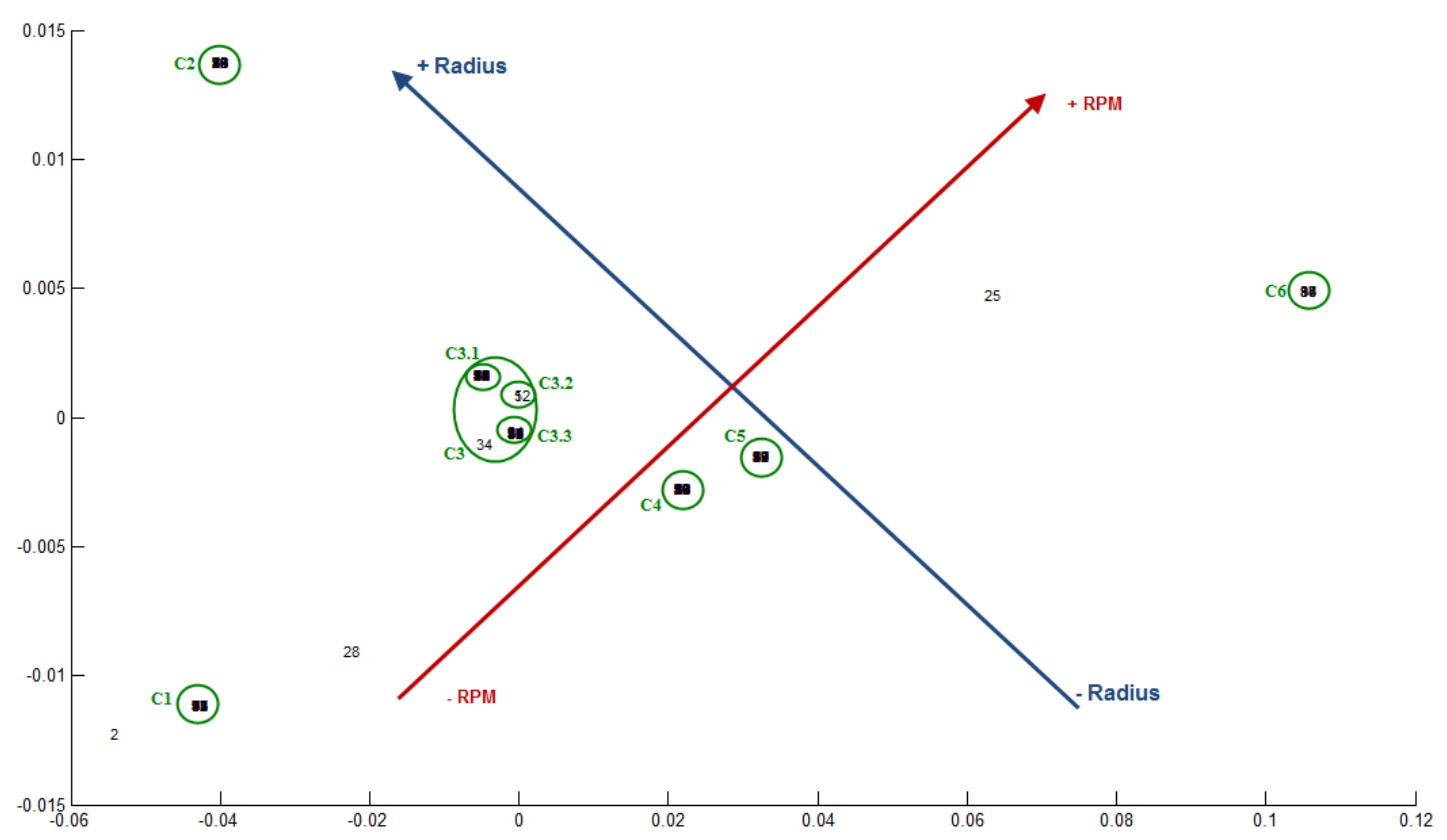

Fig. 8.a Projection of PCA

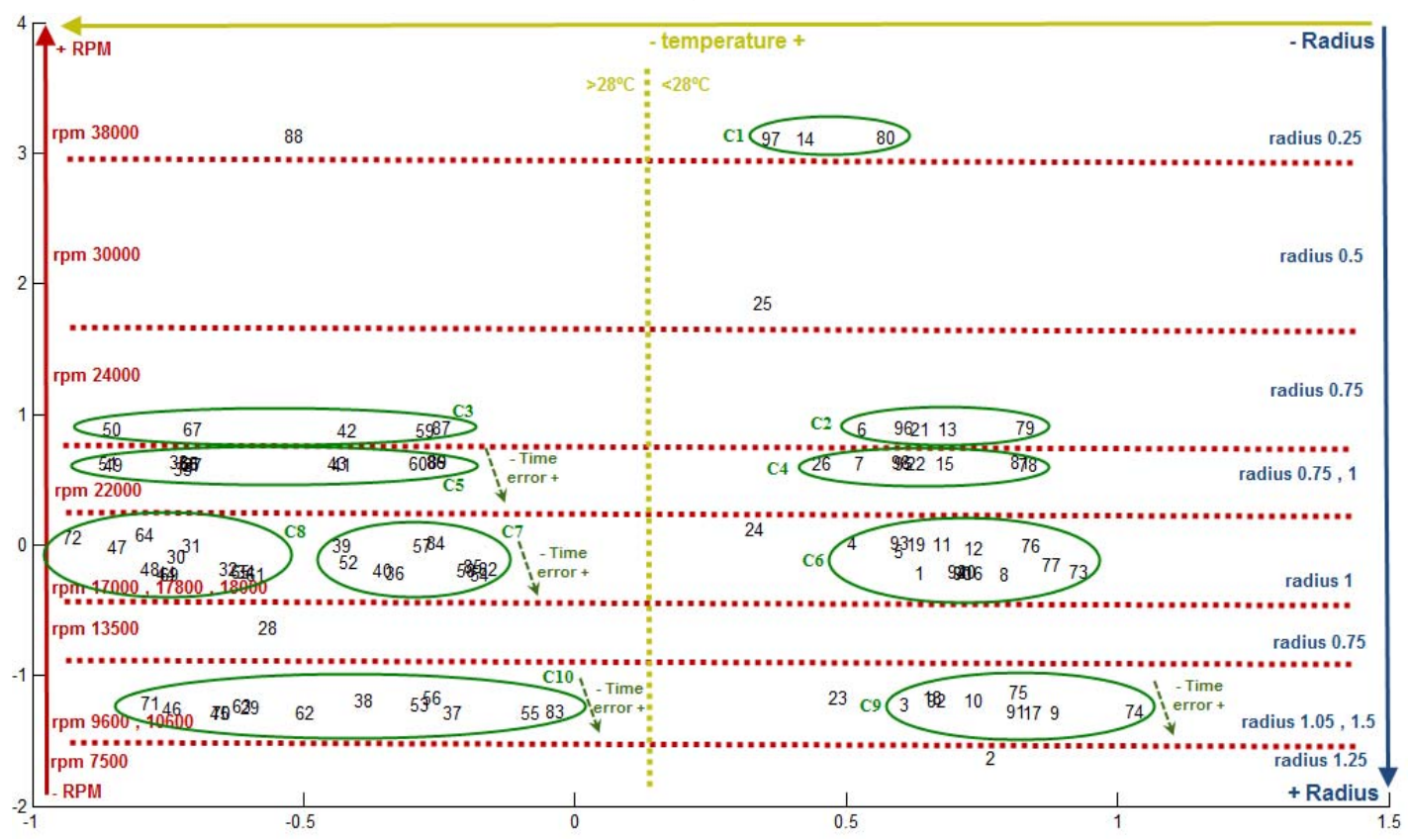

Fig. 8.b CMLHL projection after 100,000 iterations using a learning rate of $0.01, p=0.5$ and $\tau=0.05$

Fig. 8 PCA projection (Fig. 8.a) and CMLHL projection (Fig. 8.b)

\begin{tabular}{|c|c|c|c|c|}
\hline \multicolumn{2}{|c|}{ Cluster } & Samples & RPM & Radius \\
\hline \multicolumn{2}{|l|}{ - } & 2 & 7,500 & 1.25 \\
\hline \multicolumn{2}{|l|}{ C1 } & $9,17,37,45,53,55,62,70,74,83,91$ & 9,600 & 1.05 \\
\hline \multicolumn{2}{|l|}{ C2 } & $3,10,18,23,29,38,46,56,63,71,75,92$ & 10,600 & 1.5 \\
\hline \multicolumn{2}{|l|}{ - } & 28 & 13,500 & 0.75 \\
\hline \multirow{4}{*}{ C3 } & C3.1 & $\begin{array}{l}1,8,16,20,30,32,36,40,44,48,52,54,58,61,65 \text {, } \\
69,73,77,82,85,90,94\end{array}$ & 17,000 & \multirow{4}{*}{1} \\
\hline & & 34 & 17,000 & \\
\hline & C3.2 & 5,12 & 18,000 & \\
\hline & C3.3 & $4,11,19,24,31,39,47,57,64,72,84,93$ & 17,800 & \\
\hline
\end{tabular}




\begin{tabular}{|l|l|l|l|}
\hline \multirow{3}{*}{ C4 } & $\begin{array}{l}7,15,22,26,27,33,41,43,49,51,60,66,68,86,89, \\
95,98\end{array}$ & \multirow{2}{*}{22,000} & 0.75 \\
\cline { 2 - 2 } \cline { 4 - 4 } & 1 & 24,000 & 0.75 \\
\hline C5 & $6,13,21,42,50,59,67,79,87,96$ & 30,000 & 0.5 \\
\hline- & 25 & 38,000 & 0.25 \\
\hline C6 & $14,80,88,97$ & & \\
\hline
\end{tabular}

Table 2 Samples description and clusters obtained by using PCA method

\begin{tabular}{|c|c|c|c|c|}
\hline Cluster & Samples & RPM & Radius & Temperature \\
\hline C1 & $80,14,97$ & 38,000 & 0.25 & 24.1 to 25.3 \\
\hline- & 88 & 38,000 & 0.25 & 28.4 \\
\hline- & 25 & 30,000 & 0.5 & 25.7 \\
\hline $\mathrm{C} 2$ & $79,13,21,6,96$ & 24,000 & 0.75 & 24.1 to 25.3 \\
\hline $\mathrm{C3}$ & $87,59,42,67,50$ & 24,000 & 0.75 & 28.4 to 31 \\
\hline $\mathrm{C4}$ & $78,81,15,22,7,95,98,26$ & 22,000 & 0.75 & 24.1 to 25.7 \\
\hline C5 & $86,89,60,41,43,27,33,35,66,68,49,51$ & 22,000 & $0.75,1$ & 28.4 to 31 \\
\hline C6 & $73,77,76,8,11,12,16,20,19,90,94,1,4,93,5$ & $\begin{array}{l}17,000, \\
17,800, \\
18,000\end{array}$ & 1 & 24.1 to 25.3 \\
\hline- & 24 & 17,800 & 1 & 25.7 \\
\hline C7 & $82,85,84,54,58,57,52,36,40,39$ & $\begin{array}{l}17,000 \\
17,800\end{array}$ & 1 & 28.4 to 29.3 \\
\hline $\mathbf{C 8}$ & $30,32,61,65,31,64,44,48,69,47,72$ & $\begin{array}{l}17,000 \\
17,800\end{array}$ & 1 & $30.4,31$ \\
\hline- & 28 & 13,500 & 0.75 & 30.4 \\
\hline C9 & $74,75,9,10,17,18,91,3,92$ & $\begin{array}{l}9,600, \\
10,600\end{array}$ & $\begin{array}{l}1.05, \\
1.5\end{array}$ & 24.1 to 25.3 \\
\hline- & 23 & 10,600 & 1.5 & 25.7 \\
\hline C10 & $83,55,56,53,37,38,62,29,63,45,70,46,71$ & $\begin{array}{l}9,600, \\
10,600\end{array}$ & $\begin{array}{l}1.05, \\
1.5\end{array}$ & 28.4 to 31 \\
\hline- & 2 & 7,500 & 1.25 & \\
\hline
\end{tabular}

Table 3 Samples description and clusters obtained by using CMLHL method

When the dataset is considered sufficiently informative, as in this case, the next step is to model the relations between inputs and production time errors in the process, which is begun by applying several artificial neural network modelling systems.

A multilayer perceptron network (feedforward network) was used to monitor time error detection in the manufacturing of dental pieces. Data set is pre-processed from the input and output normalization step (normalizing the minimum and maximum values to $[-11]$ ), the reduction of the input vectors dimension (the data set gathered in the previous step). ANN is trained from the most widely used training algorithms such as the Lenvenberg-Marquardt algorithm [74], quasi-Newton methods [75], the resilient backpropagation algorithm [76] and the escalated conjugate gradient algorithm [77], using criteria from early stopping and Bayesian regularization techniques [78]. 
The graphic representations of the prediction $\left(\hat{y}_{1}(t \mid m)\right)$ of time error detection in the manufacturing of dental pieces versus the real time measured $\left(y_{1}(t)\right)$ for the model chosen are shown in Fig. 9. These figures were used to validate the models. In Fig. 9.a and Fig. 9.b the X-axis shows the total number of samples. In Fig. 9.a and Fig. 9.b the Y-axis represents the normalized output and unnormalised output variable range, respectively, which refers to the time errors for manufacturing.

Table 4 shows the features for the best ANN proposed: the characteristics and qualities for estimation and prediction, and its indexes (indicator values). The final model chosen is a Feedforward Network. The ANN structure has 30 hyperbolic tangent units (layer 1), 20 hidden hyperbolic tangent units (layer 2), 5 hidden hyperbolic tangent units (layer 3 ) and 1 linear output unit. The network is estimated by using the Lenvenberg-Marquardt algorithm with Bayesian regularized criterion. This model does not only present a lower loss function (V) and error values (NSSE), but also a higher system representation index value FIT1. Also a good FIT value and a small variance of the mean square errors $(\lambda)$.

From Fig. 9, it may be concluded that the ANN selected is able to simulate and predict the behaviour of time errors for the manufacturing of dental pieces (as a consequence of the production process). They are capable of modelling more than $86 \%$ of the actual measurements.

\begin{tabular}{|c|c|}
\hline Model & Indexes \\
\hline $\begin{array}{l}\text { The feedforward network has } 30 \text { hyperbolic tangent units (layer 1), } 20 \text { hidden hyperbolic } \\
\text { tangent units (layer 2), } 5 \text { hidden hyperbolic tangent units (layer } 3 \text { ) and } 1 \text { linear output } \\
\text { unit [30 } 205 \text { 1]. The network is estimated using the resilient back-propagation algorithm } \\
\text { with early stopping criterion. }\end{array}$ & $\begin{array}{l}\text { FIT1: } 71.03 \% ; \mathrm{V}: 0.024 \\
\text { NSSE: } 0.033 ; \\
\lambda: 0.00023 ; \text { FIT: } 85.19 \%\end{array}$ \\
\hline $\begin{array}{l}\text { The feedforward network has } 30 \text { hyperbolic tangent units (layer 1), } 25 \text { hidden hyperbolic } \\
\text { tangent units (layer 2), } 25 \text { hidden hyperbolic tangent units (layer 3), } 4 \text { hidden hyperbolic } \\
\text { tangent units (layer 4) and } 1 \text { linear output unit [30 } 25254 \text { 1]. The network is estimated } \\
\text { using the resilient backpropagation algorithm with early stopping criterion. }\end{array}$ & $\begin{array}{l}\text { FIT1: } 73.00 \% ; \mathrm{V}: 0.017 \\
\text { NSSE: } 0.038 ; \\
\lambda: 0.00007 ; \text { FIT: } 87.43 \%\end{array}$ \\
\hline 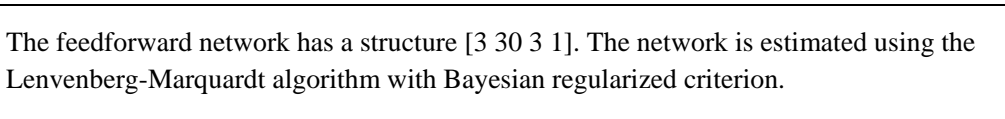 & $\begin{array}{l}\text { FIT1: } 74.36 \% ; \mathrm{V}: 0.018 ; \\
\text { NSSE: } 0.019 ; \\
\lambda: 0.00042 ; \text { FIT: } 86.45 \%\end{array}$ \\
\hline $\begin{array}{l}\text { The feedforward network has a structure [30 } 205 \text { 1]. The network is estimated using the } \\
\text { Lenvenberg-Marquardt algorithm with Bayesian regularized criterion. }\end{array}$ & $\begin{array}{l}\text { FIT1: 78.57\%; }: 0.0097 \\
\text { NSSE: } 0.015 ; \\
\lambda: 0.000059 ; \text { FIT: } 86.87 \%\end{array}$ \\
\hline $\begin{array}{l}\text { The feedforward network has a structure [30 } 25254 \text { 1]. The network is estimated using } \\
\text { the Lenvenberg-Marquardt algorithm with Bayesian regularized criterion. }\end{array}$ & $\begin{array}{l}\text { FIT1: 75.31\%;V: 0.015; } \\
\text { NSSE: 0.0168; } \\
\lambda: 0.00012 ; \text { FIT: } 85.03 \%\end{array}$ \\
\hline $\begin{array}{l}\text { The feedforward network has a structure [30 } 2051 \text { ]. The network is estimated using the } \\
\text { quasi-Newton algorithm with early stopping criterion. }\end{array}$ & $\begin{array}{l}\text { FIT1: 71.17\%; }: 0.023 ; \\
\text { NSSE: 0.028; } \\
\lambda: 0.000054 ; \text { FIT: } 88.49 \%\end{array}$ \\
\hline $\begin{array}{l}\text { The feedforward network has a structure [30 } 2051 \text { ]. The network is estimated using the } \\
\text { escalated conjugate gradient algorithm with early stopping criterion. }\end{array}$ & $\begin{array}{l}\text { FIT1: 71.37\%;V: 0.018; } \\
\text { NSSE: 0.042; } \\
\lambda: 0.00012 ; \text { FIT: } 85.76 \%\end{array}$ \\
\hline $\begin{array}{l}\text { The feedforward network has a structure [30 } 2051 \text { ]. The network is estimated using the } \\
\text { Lenvenberg-Marquardt algorithm with early stopping criterion. }\end{array}$ & $\begin{array}{l}\text { FIT1: } 70.17 \% \text {;V: } 0.035 ; \\
\text { NSSE: 0.045; } \\
\lambda: 0.0037 \text {; FIT: } 89.39 \%\end{array}$ \\
\hline $\begin{array}{l}\text { The feedforward network has a structure [30 } 25254 \text { 1]. The network is estimated using } \\
\text { the Lenvenberg-Marquardt algorithm with early stopping criterion. }\end{array}$ & $\begin{array}{l}\text { FIT1: 77.67\%;V: 0.0098; } \\
\text { NSSE: 0.035; } \\
\lambda: 0.000045 ; \text { FIT: } 89.39 \%\end{array}$ \\
\hline
\end{tabular}

Table 4 Indicator values for several proposed models of time error for manufacturing under the Dental Milling process 


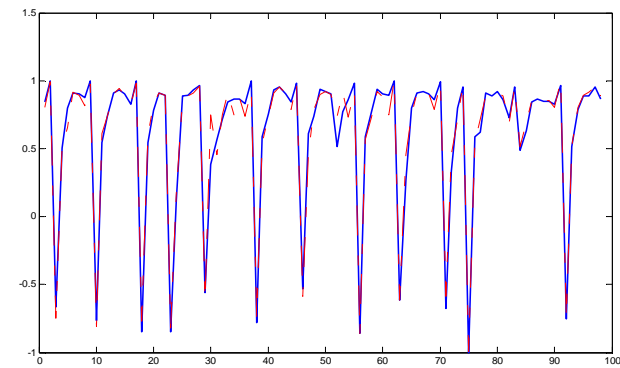

Fig. 9.a Normalized output response of the model

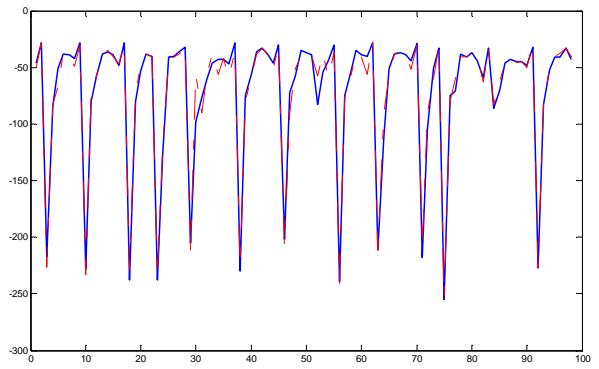

Fig. 9.b Unnormalized output response of the model

Fig. 9 Output response of the model: The feedforward network [30 205 1] is estimated using the Lenvenberg-Marquardt algorithm with Bayesian regularized criterion. The current output (solid line) is graphically presented with prediction (dash-dot line)

The model of the time error obtained may be used not only to predict time errors for the manufacture of dental pieces, but as a fitness function in the next step to determine the best operating conditions of dental milling processes. GA starts with a randomly generated initial population of size 100 individuals. Tournament selection is used to determine the parents for the next generation. Individuals from the current population are selected proportionally to their fitness, thus forming the basis for the next generation. Two-point crossover combines two parents to form a new individual for the next generation. And adaptive feasible mutation makes small changes in the individuals in the population. The population obtained by these genetic modifications is evaluated against the fitness function and enters a new search process in the next generation. The algorithm stops after it reaches a fixed number of generations and the best individual is returned as a solution to the given problem. Fig. 10 shows the output response of the time error for different unnormalised input variable ranges. In Fig. 10.a the X-axis shows the revolutions per minute (RPM), from 10,000 to 35,000 RPM. The Y-axis shows the temperature from $24^{\circ} \mathrm{C}$ to $31^{\circ} \mathrm{C}$, and the Z-axis represents the unnormalised output variable range from -200 s to $200 \mathrm{~s}$ (seconds). The time error is also shown on the bar. In Fig. 10.b the X-axis shows the temperature from $24^{\circ} \mathrm{C}$ to $31^{\circ} \mathrm{C}$, and the Y-axis represents the unnormalised output variable range, from $-100 \mathrm{~s}$ to $60 \mathrm{~s}$ for a constant value of 20,000 RPM. In both figures the radius is fixed to a constant value of $0.75 \mathrm{~mm}$. In Fig. 10.c the X-axis shows the radius, from $0.25 \mathrm{~mm}$. to $1.5 \mathrm{~mm}$. The $\mathrm{Y}$-axis shows the temperature from $24^{\circ} \mathrm{C}$ to $31{ }^{\circ} \mathrm{C}$ and the Z-axis represents the unnormalised output variable range from $-80 \mathrm{~s}$ to $20 \mathrm{~s}$. The time error is shown on the bar, too. In Fig. 10.d the X-axis shows the temperature from $24^{\circ} \mathrm{C}$ to $31^{\circ} \mathrm{C}$, and the $\mathrm{Y}$-axis represents the unnormalised output variable range, from $-30 \mathrm{~s}$ to $5 \mathrm{~s}$ for a constant radius value of $1 \mathrm{~mm}$. In both figures the number of revolutions is fixed per minute to a constant value of 30,000 RPM.

Some results obtained in order to obtain the best optimisation of the time errors for different conditions of operation fixed are shown following. For example, the time error can be optimised for different values of radius, temperature and RPM; i.e., it is possible to achieve a time error close to zero for a radius of 1.45 $\mathrm{mm}, 22,834 \mathrm{RPM}$ and a temperature of $27.69^{\circ} \mathrm{C}$. Furthermore, if the temperature is fixed to $26^{\circ} \mathrm{C}$ and the time error is close to zero, the revolutions and the radius to optimise those variables are 37,592 RPM and $1 \mathrm{~mm}$., respectively.

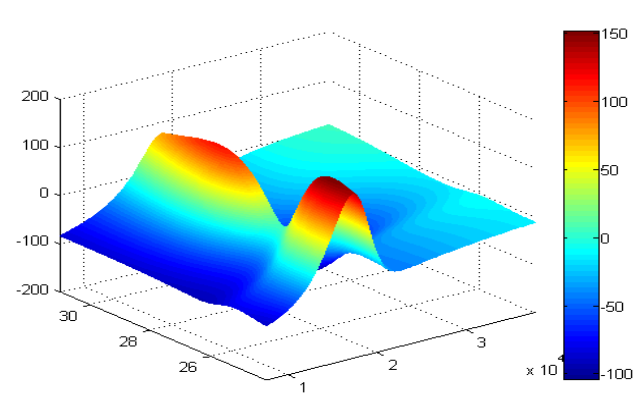

Fig. 10.a 3D graph, the $X$-axis represents the RPM, the $Y$-axis the temperature and the $\mathrm{Z}$-axis the output (time error). The other variable radius is fixed to $0.75 \mathrm{~mm}$

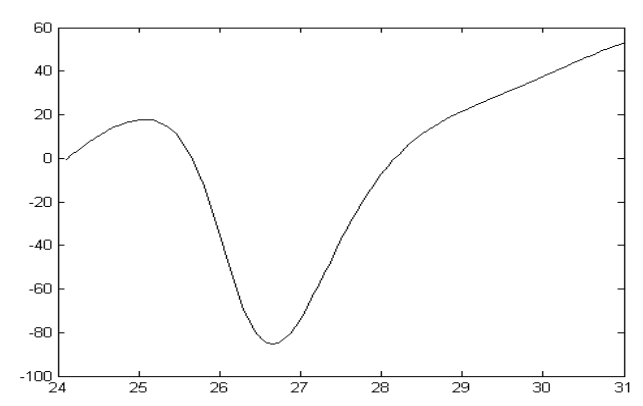

Fig. 10.b 2D graph, the X-axis represents the temperature and the Y-axis the output (time error). The others variables, RPM and radius are fixed to 20,000 RPM and 0,75 mm, respectivily 


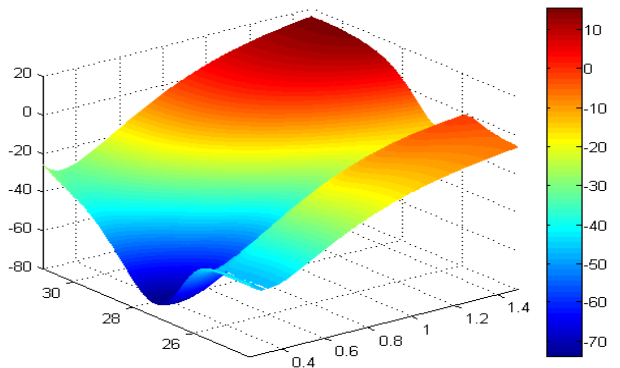

Fig. 10.c 3D graph, the $\mathrm{X}$-axis represents de radius, de $\mathrm{Y}$-axis the temperature and the $\mathrm{Z}$-axis the output (time error). The other variable, RPM is fixed to 30,000 RPM

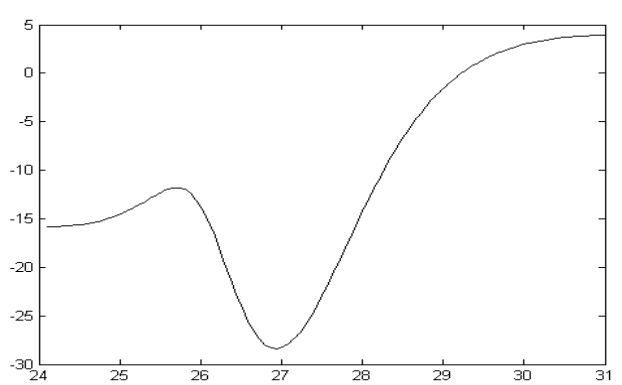

Fig. 10.d 2D graph, the $X$-axis represents the temperature and the Y-axis the output (time error).The others variables, radius and RPM are fixed to $1 \mathrm{~mm}$ and 30,000 RPM, respectivily

Fig. 10 Output response of the time error for different unnormalised input variable ranges

\section{Conclusions and future work}

The novel soft computing optimisation process described in this study can be used to optimise machine parameters for industrial processes, based on the obtained results. This method increases the companies' efficiency and substantially reduces the cost of preparing and setting machine processes. It also helps in the production process using new materials. We have used this method for optimisation and adjustments during the manufacturing process of dental pieces such as implants according to medical specifications for precise mouldings.

The method proposed is based on the selection of the most important features in an initial step. ANN are then used for modelling the features. Finally, a GA tries to achieve the best conditions for manufacturing from the model. The ANN model is used as fitness function in the GA.

The dental milling process presents an important manufacturing time error rate of about $29 \%$. This is due to the difference between the estimated time of the machine itself and the real production time. The obtained model is capable of modelling more than $86 \%$ of the actual measurements in relation to time error (modelling more than $96.8 \%$ of real time work). This helps to reduce the error and the variability rate of manufacturing processes down to $4 \%$, compared to the initial $29 \%$, which is an acceptable error rate in planning work for dental milling.

Future lines of research include modelling the temperature difference and the erosion difference (difference between diameters of the tool before and after the manufacturing), which helps to measure the accuracy of the dental milling process. Additionally, it will investigate the selection of the most suitable features using a wrapper feature selection method, in which genetic algorithms and neural networks are hybridized. Finally, an algorithm will be developed to automatically identify the best operating conditions: minor time errors for the manufacturing of dental pieces and minor erosion. The resulting model would moreover be applied to different metals used in prosthetic dentistry and in other industrial processes, such as the automotive sector.

Acknowledgments This research is partially supported through projects of the Spanish Ministry of Economy and Competitiveness [ref: TIN2010-21272-C02-01 (funded by the European Regional Development Fund), PID 560300-200911 and TIN2008-06681-C06-04] and Junta de Castilla y Leon [exp: CCTT/10/BU/0002]. The authors would also like to thank to ESTUDIO PREVIO (Madrid-Spain) for its collaboration in this research. This work was also supported in the framework of the IT4 Innovations Centre of Excellence project, reg. no. CZ.1.05/1.1.00/02.0070 by operational programme 'Research and Development for Innovations' funded by the Structural Funds of the European Union and state budget of the Czech Republic, EU.

\section{References}

[1] M.A. Fuster-Torres, S. Albalat-Estela, M. Alcañiz-Raya, M. Peñarrocha-Diago, CAD / CAM dental systems in implant dentistry: Update. Med Oral Patol Oral Cir Bucal. 2009 Mar 1; 14 (3):E141-5 
[2] T. Takahashi, J. Gunne, Fit of implant frameworks: An in vitro comparison between two fabrication techniques, J Prosthet Dent, 89, 2003, pp. 256-260

[3]A. Ortorp, T. Jemt, T. Back, T. Jalevik, Comparisons of precision of fit between cast and CNC-milled titanium implant frameworks for the edentulous mandible. Int J Prosthodont, 16, 2003, pp. 194-200

[4] S.A. Al-Fadda, G.A. Zarb, Y. Finer, A comparison of the accuracy of fit of 2 methods for fabricating implantprosthodontic frameworks. Int J Prosthodont, 20, 2007, pp. 125-131

[5] M. Romeo, J. Vallejo, J.A. Martínez, J. del Río, J. López-Quiles, M.A. Rivero, E. Pozuelo,. Tecnología CAD/CAM en implantoprótesis. Puesta al día y perspectivas de futuro, Gaceta Dental: Industria y Profesiones, 2010 JUL; XXI (216)

[6] F. Beuer, J. Schweiger, D. Edelhoff, Digital dentistry: an overview of recent developments for CAD/CAM generated restorations, Br Dent J., 204(9), May 10, 2008; pp. 497-502

[7] JC Wu, LC Lai. CG Sheets, J Earthman, R Newcomb, A comparison of the marginal adaptation of cathode-arc vapordeposited titanium and cast base metal copings, Journal of prosthetic dentistry, Volume 105, Issue 6, June 2011, pp. 403409

[8] V. Vera, A.E. Garcia, M.J. Suarez, B. Hernando, R. Redondo, E. Corchado, M.A. Sanchez, A. Gil, J. Sedano, Optimizing a dental milling process by means of Soft Computing Techniques, 10th International Conference on Intelligent Systems Design and Applications (ISDA 2010), 978-1-4244-8134-7, IEEE, 2010, pp. 1430-1435

[9] V. Vera, A.E. Garcia, M.J. Suarez, B. Hernando, E. Corchado, M.A. Sanchez, A. Gil, R. Redondo, J. Sedano, A bioinspired computational high-precision dental milling system, Proceedings of the World Congress on Nature and Biologically Inspired Computing (NaBIC2010), 978-1-4244-7377-9, IEEE, 2010, pp. 423-429

[10] O. Cordón, A. Fernández-Caballero, J. A. Gámez, F. Hoffmann, The impact of soft computing for the progress of artificial intelligence, Applied Soft Computing, Volume 11, Number 2, March 2011, pp. 1491-1492

[11] L. Ljung. System Identification, Theory for the User, Prentice-Hall, Upper Saddle River, N.J., USA, 2nd edition, 1999

[12] J. Sedano, E. Corchado, L. Curiel et al, The application of a two-step AI model to an automated pneumatic drilling process, International Journal of Computer Mathematics, Vol. 86, No. 10-11, 2009, pp. 1769-1777

[13] B. Ozcelik, A. Ozbay, E. Demirbas, Influence of injection parameters and mold materials on mechanical properties of ABS in plastic injection molding, International Communications in Heat and Mass transfer, 37(9), Nov 2010, pp.13591365

[14] V. Kumar, Jaimal Singh Khamba, Statistical analysis of experimental parameters in ultrasonic machining of tungsten carbide using the Taguchi approach, Journal of the American Ceramic Society, 91(1), Jan 2008, pp. 92-96

[15] J. Li, G.K. Ananthasuresh, A quality study on the excimer laser micromachining of electro-thermal-compliant micro devices, Journal of Micromechanics and microengineering, 11(1), Jan 2001, pp. 38-47

[16] Hsu-Hwa Chang, Yan-Kwang Chen, Neuro-genetic approach to optimize parameter design of dynamic multiresponse experiments, Applied Soft Computing, 11(1), Jan 2011, pp. 436-442

[17] P. Leray, P. Gallinari, Feature selection with neural networks, Behaviormetrika, Vol. 26, January 1999, pp. 145-166

[18] A. Verikas, M. Bacauskiene, Feature selection with neural networks, Pattern Recognition Letters, Volume 23, Issue 11, September 2002, pp. 1323-1335

[19] S. Fujita, Retrieval parameter optimization using genetic algorithms, Inf. Process, Manage, 45, Nov 2009, pp.664-682

[20] A.L.I. Oliveira, P.L. Braga, R.M.F. Lima, M.L Cornélio, Ga-based method for feature selection and parameters optimization for machine learning regression applied to software effort estimation, Inf. Softw. Technol., 52, Nov 2010, pp. 1155-1166.

[21] P. Palanisamy, I. Rajendra, S. Shanmugasundaram, Optimization of machining parameters using genetic algorithm and experimental validation for end-milling operations, The International Journal of Advanced Manufacturing Technology, 32, 2007, pp. 644-655

[22] K. Pearson, On lines and planes of closest fit to systems of points in space, Philosophical Magazine, 1901, 2(6):559572

[23] H. Hotelling, Analysis of a complex of statistical variables into Principal Components. Journal of Education Psychology, 24, 1933, pp. 417-444

[24] E. Oja, H. Ogawa, J. Wangviwattana, Principal Components Analysis by Homogeneous Neural Networks, part 1, The Weighted Subspace Criterion, IEICE Transaction on Information and Systems, E75D, 1992,pp. 366-375

[25] E. Corchado, C. Fyfe, Connectionist Techniques for the Identification and Suppression of Interfering Underlying Factors, Int. Journal of Pattern Recognition and Artificial Intelligence, 17(8), 2003, pp. 1447-1466.

[26] I. Rojas, O. Valenzuela, F. Rojas, A. Guillén, L. J. Herrera, H. Pomares, L. Marquez, M. Pasadas, Soft-computing techniques and ARMA model for time series prediction, Neurocomputing, Volume 71, Numbers 4-6, January 2008, pp. 519-537

[27] Y. Liu, C. Zhou, D. Guo, K. Wang, W. Pang, Y. Zhai, A decision support system using soft computing for modern international container transportation services, Applied Soft Computing, Volume 10, Number 4, September 2010, pp. 1087-1095

[28] A. Abraham, Hybrid Soft Computing and Applications, International Journal of Computational Intelligence and Applications, World Scientific Press, Singapore, Vol. 8, No. 1, 2009, pp. 5-7

[29] T. Wilk, M. Wozniak: Soft computing methods applied to combination of one-class classifiers, Neurocomputing, Volume 75, Issue 1, January 2012, pp 185-193

[30] S. García, J. Derrac, J. Luengo, C. J. Carmona, F. Herrera: Evolutionary selection of hyperrectangles in nested generalized exemplar learning, Applied. Soft Computing, Volume 11 Number 3, 2011, pp. 3032-3045

[31] T. Kohonen, The self-organizing map, Neurocomputing, 21(1-3), 1998, pp. 1-6

[32] E. Corchado, B. Baruque: WeVoS-ViSOM: An ensemble summarization algorithm for enhanced data visualization, Neurocomputing, Volume 75, Issue 1, January 2012, pp 171-184

[33] J. Sedano, E. de la Cal, L. Curiel, J.R. Villar, E. Corchado, Soft Computing for detecting thermal insulation failures in buildings, Proceedings of the 9thInternational Conference on Computational and Mathematical Methods in Science and Engineering, CMMSE2009, Vol. 4, 2009, pp. 1392-1402 
[34] J. Sedano, L. Curiel, E. Corchado, E. de la Cal, J.R. Villar, A Soft Computing Based Method for Detecting Lifetime Building Thermal Insulation Failures, Integrated Computer-Aided Engineering, IOS Press 17(2), 2010, pp. 103-115.

[35] P. Diaconis, D. Freedman, Asymptotics of Graphical Projections, The Annals of Statistics, 12(3), 1984, pp. 793-815

[36] E. Corchado, D. MacDonald, C. Fyfe, Maximum and Minimum Likelihood Hebbian Learning for Exploratory Projection Pursuit, Data Mining and Knowledge Discovery, 8(3), 2004, pp. 203-225

[37] J.H. Friedman, Exploratory Projection Pursuit, Journal of the American Statistical Association, Vol. 82, No. 397, Mar. 1987, pp. 249-266

[38] J.H. Freedman, J.W. Tukey, Projection Pursuit algorithm for Exploratory data-analysis, IEEE Transactions on Computers, 23(9), 1974, pp. 881-890

[39] I. Guyon, A. Elisseeff, An introduction to variable and feature selection, Journal of Machine Learning Research, Special Issue on variable and Feature Selection, 3, 2003, pp. 1157-1182

[40] H. Liu, L. Yu, Toward integrating feature selection algorithms for classification and clustering, IEEE Knowledge and Data Engineering, IEEE Transactions, 17(4), 2005, pp. 491-502

[41] Q. He, Z. Xie, Q. Hu, C. Wu, Neighborhood based sample and feature selection for SVM classification learning, Neurocomputing, Volume 74, Number 10, May 2011, pp. 1585-1594

[42] A. R. Teixeira, A. M. Tomé, E. W. Lang, Unsupervised feature extraction via kernel subspace techniques, Neurocomputing, Volume 74, Number 5, February 2011, pp. 820-830

[43] Z. Lai, M. Wan, Z. Jin, J. Yang, Sparse two-dimensional local discriminant projections for feature extraction, Neurocomputing, Volume 74, Number 4, January 2011, pp. 629-637

[44] Y. Zhang, P. Rockett, A generic optimising feature extraction method using multiobjective genetic programming, Applied Soft Computing, Volume 11, Number 1, January 2011, pp. 1087-1097

[45] L. G. Sánchez Giraldo, G. Castellanos-Domínguez, Weighted feature extraction with a functional data extension, Neurocomputing, Volume 73, Numbers 10-12, June 2010, pp. 1760-1773

[46] E. Corchado, A. Herrero, Neural visualization of network traffic data for intrusion detection, Applied Soft Computing, 11(2), 2011, pp. 2042-2056

[47] A. Herrero, E. Corchado, P. Gastaldo, R. Zunino, Neural projection techniques for the visual inspection of network traffic, Neurocomputing, 72(16-18), 2009, pp. 3649-3658

[48] H. Seung, N. Socci, D. Lee, The Rectified Gaussian Distribution, Advances in Neural Information Processing Systems 10, 1998, pp. 350-356

[49] E. Corchado, Y. Han, C. Fyfe, Structuring global responses of local filters using lateral connections, Journal of Experimental \& Theoretical Artificial Intelligence 15(4), 2003, pp. 473-487

[50] M. Nørgaard, O. Ravn, N.K. Poulsen, L.K Hanse, Neural Networks for Modelling and Control of Dynamic Systems, Springer-Verlag, 2000, London U.K.

[51] P. Stoica, T. Söderström, A useful parametrization for optimal experimental design, In IEEE Trans. Automatic. Control, AC-27, 1982

[52] J. Schoukens, Y. Rolain, R. Pintelan, Improved approximate identification of nonlinear systems, In $21^{\text {st }}$ IEEE Instrumentation and Measurement Technology Conference, Como, Italy, 2004, pp. 2183-2186

[53] A. Azadeh, Z. S. Faiz, A meta-heuristic framework for forecasting household electricity consumption, Applied Soft Computing, Volume 11, Number 1, January 2011, pp. 614-620

[54] I. Mukherjee, P. Kumar Ray, Optimal process design of two-stage multiple responses grinding processes using desirability functions and metaheuristic technique, Applied Soft Computing, Volume 8, Number 1, January 2008, pp. 402-421

[55] F. Hayes-Roth, Review of "Adaptation in Natural and Artificial Systems by John H. Holland", The U. of Michigan Press, SIGART Bull., 53, August 1975, 15-15

[56] M. Cámara, J. Ortega, F. de Toro, A single front genetic algorithm for parallel multi-objective optimization in dynamic environments, Neurocomputing, Volume 72, Numbers 16-18, October 2009, pp. 3570-3579

[57] X.P. Zeng, Y.M. Li, J. Qin, A dynamic chain-like agent genetic algorithm for global numerical optimization and feature selection, Neurocomputing, Volume 72, Numbers 4-6, January 2009, pp. 1214-1228

[58] S. Kirkpatrick, C.D. Gelatt Jr, M.P Vecchi, Optimization by simulated annealing. Science, 220, 1983, pp. :671-680

[59] W.C. Hong, Traffic flow forecasting by seasonal SVR with chaotic simulated annealing algorithm, Neurocomputing, Volume 74, Numbers 12-13, June 2011, pp. 2096-2107

[60] F. Glover, M. Laguna, Tabu Search, Kluwer Academic Publishers, Norwell, MA, 1997, USA.

[61] B. Bolat, P. Cortés, Genetic and tabu search approaches for optimizing the hall call - Car allocation problem in elevator group systems, Applied Soft Computing, Volume 11, Number 2, March 2011, pp. 1792-1800

[62] M. Dorigo, T. Stützle, Ant Colony Optimization, Bradford Co., Scituate, MA, 2004, USA.

[63] R.A. Aliev, R.R. Aliev, B. Guirimov, K. Uyar, Dynamic data mining technique for rules extraction in a process of battery charging, Applied Soft Computing, June 2008, pp. 1252-1258

[64] I. Chaudhry, P. Drake, Minimizing total tardiness for the machine scheduling and worker assignment problems in identical parallel machines using genetic algorithms, The International Journal of Advanced Manufacturing Technology, 42, 2009, pp. 581-594

[65] A.J. Raigrodski, Contemporary materials and technologies for allceramic fixed partial dentures: a review of the literature. J Prosthet Dent. 92, 2004, pp. 557-62

[66] T. Kapos, L.M. Ashy, G.O. Gallucci, H.P. Weber, D. Wismeijer. Int J Oral Maxillofac Implants, 24, 2009, Suppl:110-7

[67] J.W. McLean, J.A. von Fraunhofer, The estimation of cement film thickness by an in vivo technique. Br Dent J 1971; $131-138$

[68] D.M. Hecker, S.E. Eckert, Cyclic loading of implant-supported protheses: Changes in component fit over time. J Prosthet Dent, 89 (4), 2003; pp. 346-351

[69] P.I. Branemark, Osseointegration and its experimental background. J Prosthet Dent 50, 1983, pp. 399-410

[70] T. Kallus, C. Bessing, Loose gold screws frequently occur in full-arch prostheses supported by osseointegrated implants after 5 years. Int J Oral Maxillofac Impl 9, 1994, pp. 169-78 
[71] H. Demuth, M. Beale, M. Hagan, Neural Network Toolbox User’s Guide, The Mathworks, Inc. 2010

[72] J. Sedano, E. Corchado, L. Curiel, J. R. Villar, E. de la Cal, Detection of heat flux failures in building using a soft computing diagnostic system, Neural network world, 20(7), 2010, pp.883-898

[73] The Math Works. Global Optimization Toolbox. The MathWorks, Inc., URL: http://www.mathworks.com/products/global-optimization/index.html

[74] R. Fletcher, Practical Methods of Optimization, Wiley \& Sons, Chichester, UK, 2nd edition, 1987

[75] J.E. Dennis, R.B. Schnabel, Numerical Methods for Unconstrained Optimization and Nonlinear Equations, Englewood Cliffs, NJ: Prentice-Hall, 1983

[76] M. Riedmiller, H. Braun, A directive adaptive method for faster backpropagation learning: The RPROP algorithm, Proceedings for IEEE International Conference on Neural Networks, 1993.

[77] M.F. Moller, A scaled conjugate gradient algorithm for fast supervised learning, Neural Networks, Vol. 6, 1993, pp. 525-533

[78] D.J.C. Mackay, Bayesian interpolation, Neural Computation, Vol. 4, No. 3, 1992, pp. 415-447. 